\section{Damien Bonnet Jean-Michel Rauzier Patrice Bouvagnet Daniel Sidi}

\section{ADRESSES}

D. Bonnet: chef de clinique assistant. D. Sidi : professeur des universités, praticien hospitalier. Hôpital Necker-Enfants Malades, Service de cardiologie pédiatrique, 149, rue de Sèvres 75743 Paris Cedex 15, France. J.M. Rauzier: interne des Hôpitaux de Montpellier. P. Bouvagnet: directeur de recherche à l'Inserm. CRBM, ERS 0155, Cnrs, et Service de cardiologie B, Hôpital A. de Villeneuve, Montpellier, France.

\title{
Génétique des cardiopathies congénitales et des cardiopathies héréditaires non myocardiques
}

La génétique moléculaire a permis d'appréhender les cardiopathies congénitales sous un angle différent. A partir d'exemples choisis, il est possible d'illustrer la conception récente de l'hérédité mendélienne des malformations cardiaques et les difficultés du clonage positionnel d'affections souvent létales, à pénétrance incomplète et subissant de grandes variations phénotypiques inter- et intrafamiliales. L'identification des microdélétions interstitielles du chromosome 22q11 dans les cardiopathies cono-troncales a ouvert plusieurs axes de recherche depuis l'embryologie jusqu'à une réflexion éthique concernant le diagnostic prénatal. Les modèles murins d'anomalie de latéralisation du cour seront probablement à l'origine du clonage des gènes humains.

es cardiopathies congénitales sont les «orphelines» de la génétique moléculaire dans le domaine de la cardiologie pour plusieurs raisons. Tout d'abord, la mortalité importante des cardiopathies complexes jusqu'à ces quinze dernières années et les imprécisions du diagnostic avant l'ère échographique n'ont pas permis de reconnaître la fréquence de l'hérédité mendélienne de certaines de ces malformations. Par ailleurs, l'approche segmentaire des cardiopathies congénitales, consistant à décrire les connexions auriculo-ven- triculaires et ventriculo-artérielles, ainsi que les différents shunts intracardiaques, a laissé dans l'ombre les relations embryologiques entre des malformations anatomiquement différentes. Aujourd'hui, l'embryologie expérimentale a permis de rassembler en six groupes des cardiopathies procédant d'un même mécanisme d'anomalie de développement embryonnaire (TableauI). Le meilleur exemple en est l'observation de la contribution des cellules de la crête neurale rhombencéphalique au développement des gros vaisseaux de la base du cœur; en effet, leur suppression 


\section{Tableau I}

\section{CLASSIFICATION DES CARDIOPATHIES CONGÉNITALES}

Anomalies de migration des cellules de la crête neurale: cardiopathies cono-troncales

- Anomalies de la région cono-troncale

- truncus arteriosus

- tétralogie de Fallot

- atrésie pulmonaire avec CIV

- agénésie des valves pulmonaires

- CIV avec malalignement du septum conal

- certaines formes de malposition vasculaire

- Anomalie des arcs aortiques

- arc aortique droit

- interruption de crosse

- sous-clavière rétro-œsophagienne

- double arc

\section{Cardiopathies de débit}

La quantité de sang qui passe par une cavité ou un orifice en détermine le développement donc la taille

- Cardiopathies de réduction du flux

du cœur gauche

- syndrome d'hypoplasie du cœur gauche

- rétrécissement mitral congénital

- sténose sous-aortique

- sténose aortique sur valve mono ou bicuspide

- co-arctation isthmique de l'aorte

du cœur droit

- atrésie pulmonaire à septum interventriculaire intact

- sténose valvulaire pulmonaire

- Cardiopathies d'excès de flux

CIA type ostium secundum

Anomalies ciblées du développement retour veineux pulmonaire anormal

Anomalies de la matrice extracellulaire

CIV périmembraneuse et musculaire

Anomalies de développement des coussins endocardiques primitifs : canaux auriculo-ventriculaires sous toutes leurs formes

Anomalies de la boucle cardiaque primitive

- anomalies du situs viscéro-auriculaire avec ou sans cardiopathie complexe

- isomérismes atriaux

- les doubles-discordances ou transposition corrigée des gros vaisseaux

CIA : communication interauriculaire; CIV : communication interventriculaire.

Modifié d'après [2].

expérimentale conduit à des cardiopathies variées (tétralogie de Fallot, atrésie pulmonaire avec communication interventriculaire, tronc artériel commun...) intéressant la région cono-troncale [1] et permet de définir ce groupe comme celui des «cardiopathies cono-troncales » correspondant à une anomalie de développement dans un champ embryonnaire (figure 1). Cette classifi- met de définir une récurrence familiale de cardiopathie congénitale comme concordante, même si les individus atteints ont des malformations anatomiquement différentes si tant est que ces malformations appartiennent au même groupe embryonnaire. Cela est illustré sur la figure 2 par l'exemple du groupe des cardiopathies cono-troncales. Cette classification trouve ses limites dans les interactions étroites entre les diffé- rents segments du cœur en développement et l'hémodynamique fœetale; elle contribue cependant à orienter plus efficacement les enquêtes familiales et à identifier un nombre croissant de cardiopathies congénitales supposées d'origine multifactorielle comme des maladies éventuellement monogéniques [3, 4].

\section{Hérédité mendélienne des cardiopathies congénitales}

La fréquence des cardiopathies congénitales associées à des anomalies chromosomiques ou héritées sur un mode mendélien était estimée à $8 \%$. Ce chiffre est sous-évalué à la lumière des progrès conceptuels mentionnés ci-dessus et il l'est d'autant plus que la mortalité fotale des cardiopathies sévères est elle aussi sous-évaluée et que les cardiopathies asymptomatiques passent inaperçues. A côté de ces insuffisances de diagnostic, d'authentiques défauts de pénétrance viennent compliquer l'identification des formes familiales. Un modèle canin de tétralogie de Fallot, les lévriers Keeshond, illustre cet inconvénient. En effet, le mode de transmission de la cardiopathie, suspecté sur les pedigrees, était autosomique récessif et c'est l'histologie fine de la région cono-troncale chez les chiens apparemment sains qui a démontré la transmission dominante de l'affection [5]. Chez l'homme, la cardiopathie congénitale la plus récurrente est l'hypoplasie du cœur gauche. L'observation de récurrences multiples dans des familles consanguines a suggéré une hérédité autosomique récessive mais quelques observations de parents d'enfants atteints ayant un équivalent mineur de maladie obstructive du cour gauche (co-arctation, membrane sous-aortique, malformation mitrale congénitale...) sont en faveur d'un mode de transmission dominant $[6,7]$. Le mode de transmission reste donc encore obscur. S'agit-il d'une hérédité mixte avec un gène majeur déterminant un phénotype complet si les deux allèles sont mutés ? Y a-t-il un seuil de susceptibilité au-delà duquel on obtient un phénotype d'hypoplasie du cœur gauche si un ou deux allèles du gène majeur sont mutés? S'agit-il d'une 


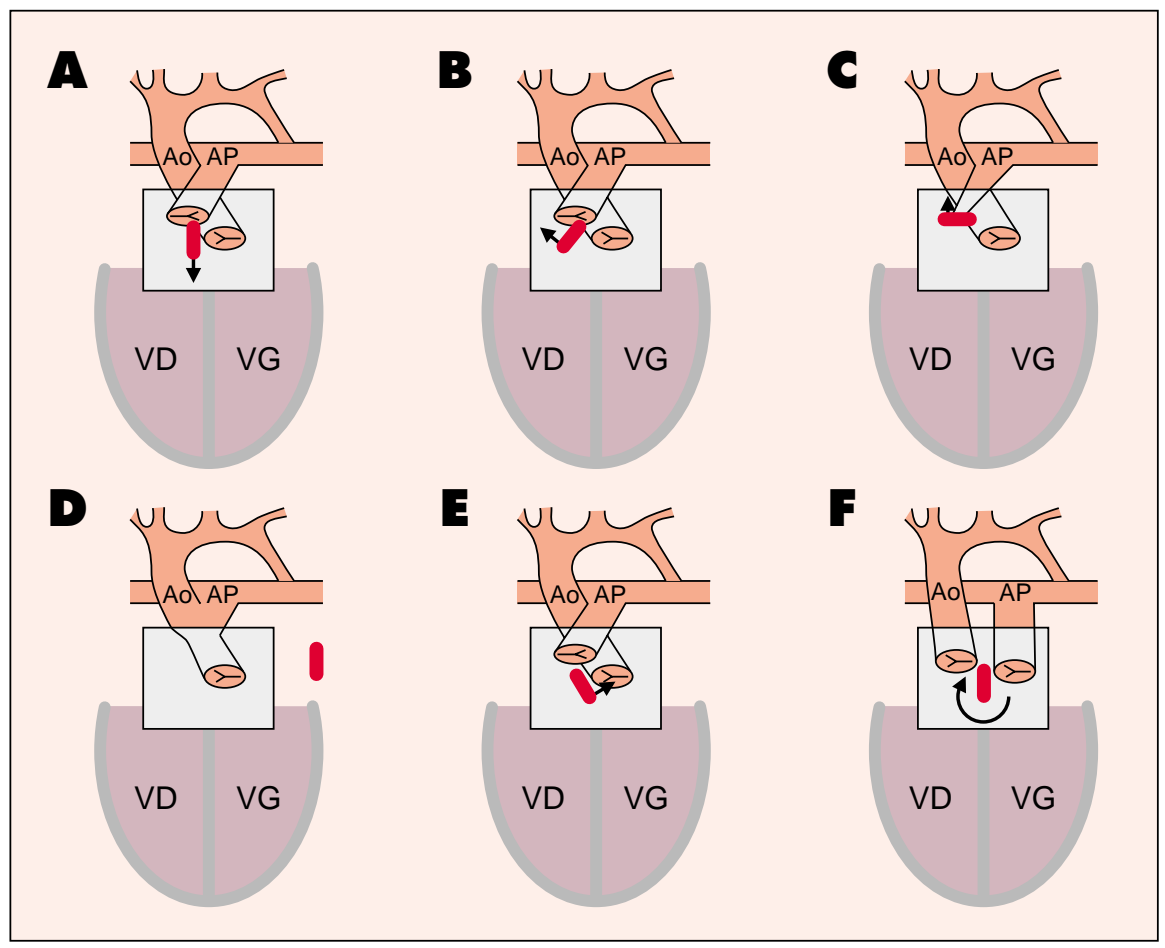

Figure 1. Cardiopathies cono-troncales. Un seul mécanisme, l'anomalie de développement du septum conal (en rouge). Au cours de la septation ventriculaire, le septum conal fusionne avec les septums musculaire et d'admission pour étanchéifier la cloison interventriculaire. Un malalignement de ce septum avec les portions sous-jacentes conduira à une communication interventriculaire. A. Septation normale. B. Bascule antérieure du septum conal avec sténose pulmonaire et communication interventriculaire: tétralogie de Fallot. C. Bascule antérieure complète du septum conal obstruant la voie pulmonaire: atrésie pulmonaire avec communication interventriculaire. D. Absence de développement du septum de la région cono-troncale: tronc artériel commun. E. Bascule postérieure du septum conal sous l'aorte: obstacle sous-aortique et interruption de la crosse aortique. F. Malrotation du septum de la région cono-troncale: transposition des gros vaisseaux. Ao: aorte ; $A P$ : artère pulmonaire ; VD : ventricule droit ; VG : ventricule gauche.

maladie multigénique? L’hémodynamique fotale influence-t-elle le phénotype différemment en fonction du nombre d'allèles mutés?

Outre la rareté des formes familiales et les difficultés rencontrées dans la collection de pedigrees informatifs nécessaires à l'établissement d'une stratégie de clonage positionnel, l'approche moléculaire des malformations cardiaques pâtit de deux autres inconvénients: la rareté des modèles animaux spontanés ou induits et le démembrement moléculaire encore balbutiant de la morphogenèse cardiaque. La conséquence de ces deux difficultés est l'absence de gènes candidats forts pour la plupart des cardiopathies malformatives héréditaires. mique dominante, associant des malformations des membres supérieurs intéressant le segment radial, un blocauriculo-ventriculaire du premier degré et une cardiopathie congénitale qui est 8 fois sur 10 une communication interauriculaire. Une grande variabilité inter- et intrafamiliale est notée à la fois pour les malformations des membres depuis l'hypoplasie de l'éminence thénar** jusqu'à la phocomélie*** et, pour les cardiopathies, allant de la communication interauriculaire ostium secundum au cœur univentriculaire. Alors que les malformations osseuses sont totalement pénétrantes bien que parfois subtiles, les cardiopathies ne sont observées que chez $85 \%$ des patients atteints [8]. Un gène du syndrome de HoltOram a pu être localisé sur le bras long du chromosome 12 par analyse de liaison $[9,10]$. L'intervalle génétique a été restreint par l'analyse d'une translocation complexe chez un foetus atteint [11] puis, par piégeage d'exons sur un contig de cosmides construit sur la région d'intérêt, le gène $T B X 5$ a été cloné et plusieurs mutations non-sens et fauxsens ont été identifiées dans des formes familiales et sporadiques de syndrome de Holt-Oram [12, 13]. La cardiopathie la plus fréquente du syndrome de Holt-Oram est la communication interauriculaire ostium secundum et plusieurs familles atteintes ont été décrites. La transmission de la cardiopathie y est autosomique dominante et, dans certains cas, un blocauriculo-ventriculaire y est associé. Cela a soulevé l'hypothèse selon laquelle ces formes familiales de communication interauriculaire seraient des phénocopies partielles du syndrome de Holt-Oram. Nous avons pu, par une cartographie d'exclusion du chromosome 12, exclure l'allélisme entre syndrome de Holt-Oram et communications interauriculaires familiales, démontrant l'hétérogénéité génétique des communications interauriculaires syndromiques et isolées [14]. Une démarche identique a permis d'exclure la «région

\footnotetext{
* Association d'une cardiopathie congénitale et de malformations squelettiques.

** Saillie faite par les muscles courts du pouce sur la paume de la main.

*** Attache des extrémités (main ou pied) directement sur le tronc.
} 


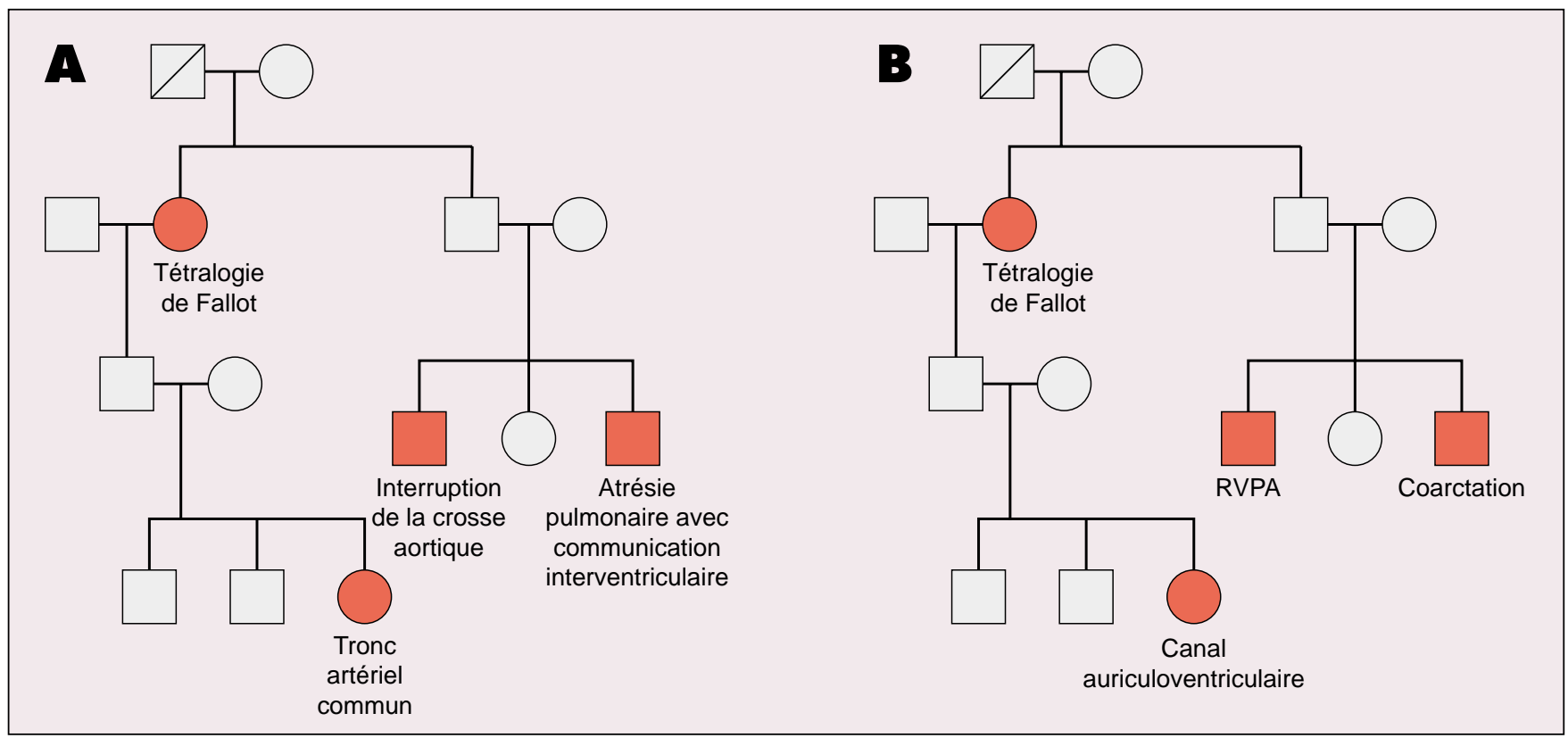

Figure 2. Cardiopathies familiales concordantes ou discordantes selon leur origine embryologique. A. Récurrence familiale concordante de cardiopathie cono-troncale appartenant au même groupe embryologique. B. Récurrence familiale "discordante" de cardiopathies a priori non liées embryologiquement. RVPA : retour veineux pulmonaire anormal.

cardiaque critique du chromosome $21 »$ contenant a priori un gène majeur du développement du canal auriculo-ventriculaire, cardiopathie élective de la trisomie 21 , dans les formes familiales non syndromiques de cette cardiopathie [15]. Cette stratégie d'identification de gènes de cardiopathies isolées passant par l'étape intermédiaire des cardiopathies syndromiques a donc été infructueuse. Un gène du syndrome de Holt-Oram a pu être localisé sur le bras long du chromosome 12 par analyse de liaison. Là encore, il s'agit d'un exemple très particulier puisque la localisation n'a été possible que grâce à une très grande famille mormone de neuf générations dans laquelle les seuls survivants atteints se trouvaient dans les deux générations les plus jeunes [16]. L'enquête épidémiologique qui a suivi a démontré un effet fondateur puisque les rares autres formes familiales de retour veineux pulmonaire anormal total liées à ce locus du chromosome 4 avaient un ancêtre commun écossais.

\section{Microdélétions et cardiopathies congénitales}

Une autre étape a été franchie grâce à l'observation d'anomalies cytogéné- tiques mineures chez des enfants ayant une cardiopathie congénitale. L'exemple le plus démonstratif est l'identification d'une microdélétion interstitielle 22q11 dans le syndrome de Di George $\left(\mathrm{m} / \mathrm{s}\right.$ 1995, $n^{\circ} 10$, p. 1500). Il s'agit d'une belle histoire encore incomplète au sein de laquelle les progrès de la biologie moléculaire ont complètement modifié la manière d'appréhender un groupe important de malformations cardiaques congénitales. DiGeorge a décrit en 1965 le syndrome qui portera son nom: hypoplasie ou aplasie des glandes parathyroïdes et du thymus, dysmorphie faciale et malformations cardiovasculaires. A. de la Chapelle (Helsinki, Finlande) fut le premier à proposer que le chromosome 22 soit impliqué dans le syndrome de DiGeorge mais les anomalies cytogénétiques du chromosome 22, ou plus rarement du chromosome 10 , n'étaient observées que chez $5 \%$ des patients [17]. C'est le mérite du groupe de Peter Scambler (Londres, Royaume-Uni) d'avoir identifié, par hybridation in situ moléculaire, une microdélétion interstitielle 22q11 chez les enfants atteints [18]. C'est cette méthode qui autorise aujourd'hui le diagnostic en routine de cette anomalie, seconde anomalie chromosomique la plus fréquente dans les cardiopathies congénitales de l'enfant après la trisomie 21. L'utilisation de marqueurs microsatellites localisés dans la région communément délétée a montré que la non contribution maternelle était un peu plus fréquente que la non-contribution paternelle dans les microdélétions 22q11 de novo qui représentent $75 \%$ des cas $[19,20]$. Plus rares, mais aux conséquences bien différentes en termes de conseil génétique et de diagnostic prénatal, sont les microdélétions 22q11 héritées d'un parent ayant la microdélétion 22q11 et transmises de façon «dominante», et les translocations équilibrées parentales intéressant le chromosome 22q11 [21]. En effet, si l'un des parents a une microdélétion $22 q 11$, il a une chance sur deux de transmettre cette microdélétion à sa descendance. Pour les translocations parentales équilibrées intéressant la région 22q11, le risque de transmission de la translocation, sous une forme déséquilibrée, est élevé. On passe donc d'un risque de récidive proche de zéro pour les microdélétions 22q11 de novo à un risque élevé en cas d'anomalie parentale. Rapidement, les syndromes apparentés au syndrome de DiGeorge - syndrome vélo-cardio-facial de Schprintzen, conotruncal anomaly face syndrom (CTAFS) et syndrome de Cay- 
ler - ont été définis comme des variantes phénotypiques d'une même affection: la microdélétion interstitielle 22q11 [22]. Dans un souci de simplification, certainement excessif et réducteur, l'acronyme CATCH 22 (cardiac defect, abnormal facies, thymic hypoplasia, cleft, hypocalcemia) a été proposé comme nouveau nom de baptême pour l'ensemble de ces syndromes $\left(m / s n^{\circ} 12,1995, p .1727\right.$; $n^{\circ} 1$, 1997, p. 109). Malheureusement, il n'embrasse pas la diversité des manifestations cliniques du syndrome et il faut, l'effet de mode aujourd'hui passé, lui préférer le terme générique de microdélétion 22q11. Les cardiopathies associées à la microdélétion 22q11 sont des cardiopathies cono-troncales (Tableau I). Ce type de malformation représente $50 \%$ des cardiopathies diagnostiquées chez le nouveau-né. La question a été immédiatement posée de savoir si les enfants ayant une cardiopathie cono-troncale isolée avaient une microdélétion 22q11, sur la base de l'hypothèse selon laquelle ils auraient un syndrome de DiGeorge très partiel dont l'expression serait limitée au cœur. La réponse a été négative; en effet, les patients ayant une microdélétion interstitielle du chromosome 22q11 ont une dysmorphie faciale caractéristique et constante diversement associée aux autres éléments précités [23]. L'hypothèse selon laquelle ces patients ont une délétion de plus petite taille a été exclue et, mieux, aucune corrélation n'a été retrouvée entre la taille de la microdélétion et le phénotype. La région communément délétée recouvre deux mégabases mais la région minimale n'est que de $300 \mathrm{~kb}$. Étant donné que les éléments phénotypiques du syndrome intéressent tous des structures dérivées des crêtes neurales rhombencéphaliques postérieures, l'hypothèse d'un gène unique gouvernant la migration de ces cellules et emporté par la microdélétion a été préférée à celle d'un syndrome de gènes contigus. Cette hypothèse était renforcée par le cas unique d'un patient ayant une translocation 2-22 apparemment équilibrée avec un point de translocation situé dans la région communément délétée. Le clonage du point de cassure n'a pas apporté de solution concluante [24] et l'observation de

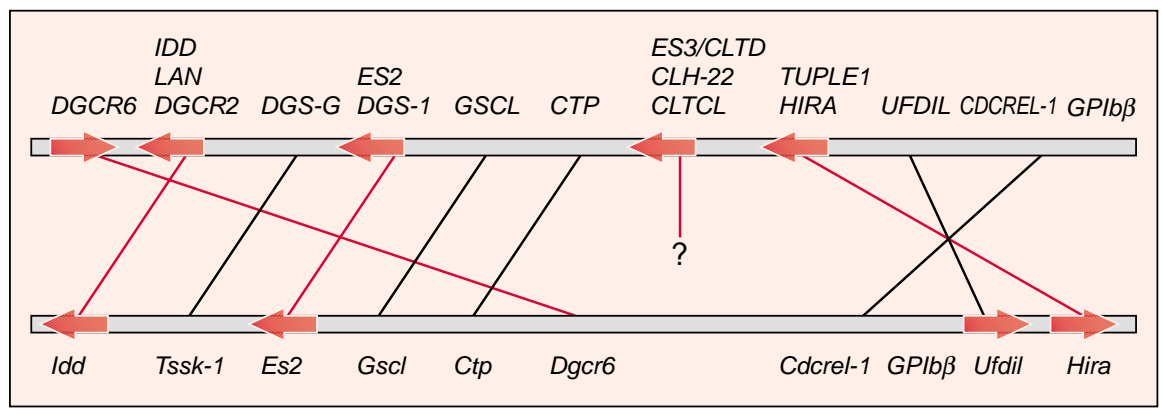

Figure 3. Organisation comparative de la région critique de délétion du chromosome 22q11 (DGCR) chez l'homme et chez la souris. Noter qu'il ne semble pas exister d'homologue murin de CLTCL. Au moins deux réarrangements chromosomiques séparent les deux espèces. Les flèches indiquent l'orientation $5^{\prime}-3^{\prime}$ des gènes lorsqu'elle est connue. L'ordre relatif des gènes Cdcrel-1 et GPIb $\beta$ n'est pas connu. Certains gènes ont été désignés par plusieurs noms qui sont superposés sur la figure. DGCR pour DiGeorge critical region, ES pour expressed sequence, HIRA pour histone transcription repressor, TUPLE-1 pour tup-like/enhancer of split gene, IDD pour integral membrane protein deleted in DiGeorge, CTP pour mitochondrial citrate transporter, CLTD pour clathrin heavy chain D, GSCL pour goosecoid like gene, GP-Ib $\beta$ pour platelet glycoprotein.

microdélétions non recouvrantes dans la région 22 q11 chez des patients au phénotype identique rend les choses plus complexes sans exclure la possibilité d'un gène unique mais en faisant intervenir un effet de position [25]. La carte physique de la région s'enrichit de jour en jour et plusieurs gènes ont été quelques temps des "candidats" (figure 3). Adoptant la même démarche que précédemment pour les communications interauriculaires et le canal auriculo-ventriculaire, une liaison en $22 q 11$ a été exclue dans les familles de cardiopathies cono-troncales isolées, nouvelle «hétérogénéité génétique" pour un groupe de cardiopathie. Mais, outre leur association aux autres éléments du syndrome de DiGeorge, les cardiopathies cono-troncales de la microdélétion 22q11 sont subtilement différentes. La distribution des différents types de cardiopathies cono-troncales dans une population de patients ayant la microdélétion est grossièrement uniforme suggérant une répartition au hasard du phénotype dans ce groupe. Cependant, si l'on compare des patients ayant une cardiopathie conotroncale avec ou sans microdélétion $22 q 11$, certaines malformations, telle que l'interruption de la crosse aortique de type $\mathrm{B}$, sont quasi spécifiques de l'anomalie moléculaire. De plus, pour un même type de malformation cono-troncale tel que la tétralogie de
Fallot, certains détails morphologiques - collatérales aorto-pulmonaires, anomalies des arcs aortiques sont l'apanage des enfants ayant la microdélétion [26]. L'hétérogénéité génétique de ces cardiopathies a été démontrée et les progrès moléculaires récents ont éclairé d'un jour nouveau les différences phénotypiques qu'on n'appréhendait que grossièrement. Ils suggèrent que les anomalies cardiaques observées dans le syndrome de DiGeorge surviennent à un moment particulier de la morphogenèse cardiaque, différent chez les patients n'ayant pas de microdélétion $22 q 11$.

L'histoire de la microdélétion interstitielle du chromosome 7q23 dans le syndrome de Williams et Beuren est le miroir de celle de la microdélétion 22q11 (m/s $n^{\circ} 3,1997, p$. 406). Elle commence par la localisation, par analyse de liaison, d'un gène responsable de sténose supravalvulaire aortique familiale transmise de façon dominante sur le bras long du chromosome 7. Quelques semaines plus tard, une translocation équilibrée entre les chromosomes 6 et 7 coségrégeant avec une sténose supravalvulaire aortique dans une famille désigne le point de cassure comme région d'intérêt [27]. Le clonage de celui-ci montre qu'il interrompt le gène codant pour l'élastine, protéine constitutive majeure de la paroi artérielle. L'identification de mutations 
ponctuelles dans le gène de l'élastine achèvera la démonstration. Le syndrome de Williams et Beuren associe une dysmorphie dite en faciès d'elfe, un retard mental avec une personnalité cocktail-party et une sténose supravalvulaire aortique. Il n'y avait qu'un pas à franchir pour rechercher une anomalie du gène de l'élastine dans ce syndrome. Et, en effet, le syndrome de Williams et Beuren est associé à une microdélétion interstitielle du chromosome 7q23 emportant tout ou partie du gène de l'élastine [28]. Contrairement à la microdélétion 22q11 dans laquelle l'ensemble des symptômes appartiennent à un même champ embryonnaire suggérant un gène unique, l'hypothèse d'un syndrome de gènes contigus était plus logique dans le syndrome de Williams et a été confirmée par des corrélations entre le phénotype partiel de certains patients, la localisation et la taille de la délétion $7 q$ [29].

\section{Modèles animaux pour des cardiopathies humaines}

Le modèle animal le plus exploité a été celui des anomalies de latéralisation ou hétérotaxie chez la souris. La morphogenèse cardiaque et la latéralisation sont intimement liées puisque la formation de la boucle cardiaque est à la fois la première étape de la morphogenèse et la première note d'asymétrie dans le cœur embryonnaire*. Les défauts de latéralisation sont des anomalies de position des organes asymétriques (cœur, estomac, foie, rate...). Il s'agit donc de perturbation de l'agencement droite/gauche de ces organes pouvant être inversés - situs inversus avec cour à droite - ou en position intermédiaire - situs ambigu. L'association à des malformations cardiaques est très fréquente et, le plus souvent, il s'agit d'une non-détermination de la latéralité intracardiaque (oreillette unique, ventricule unique, un seul vaisseau sortant du ventricule avec atrésie de l'autre vaisseau) et d'un dédoublement aléatoire d'un côté isomérisme - plutôt que d'une inversion vraie.

* Voir aussi p. 1036.
Deux modèles murins d'hétérotaxie autosomique récessive, $i v$ et $i n v$, ont été décrits. Les gènes sont localisés respectivement sur les chromosomes 12 et 4 . Une mutation faux-sens dans un gène de chaîne lourde de dynéine, lrd (left/right dynein), a récemment été identifiée chez la souris $i v$. Cette mutation intéresse un acide glutamique très conservé à la fois entre les espèces et dans les séquences des dynéines axonémales et cytoplasmiques [30]. L'homologue humain n'est pas connu mais il constituera un gène candidat majeur pour le syndrome de Kartagener, associant anomalies du situs et dyskinésie ciliaire. Le gène responsable du phénotype inv, associant hétérotaxie, anomalies des voies biliaires et dégénérescence kystique des reins, code pour une protéine contenant des motifs ankyrine dont la propriété est de lier les protéines. Là encore, le clonage de l'homologue humain permettra de rechercher des mutations chez les patients ayant un phénotype identique. La cascade de gènes intervenant dans la détermination de la boucle cardiaque commence à être démembrée (figure 4). Les hétérotaxies viscéro-auriculaires sont suspectes d'être des maladies monogéniques mais la variabilité intrafamiliale du phénotype cardiaque pourrait avoir comme origine des mécanismes aléatoires de rotation et de septation du cœur embryonnaire en l'absence du signal de latéralisation ou bien être gérée par l'intervention de plusieurs gènes

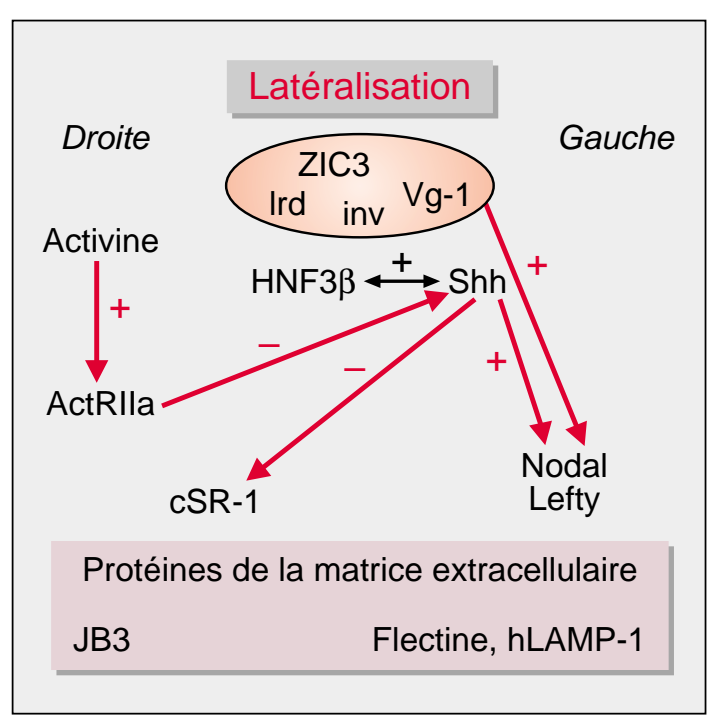

[31]. En effet, les souris doubles hétérozygotes pour les mutations nulles des gènes Nodal et $H N F-3 \beta$ ont un défaut de latéralisation alors que les simples hétérozygotes ont un phénotype normal, suggérant une hérédité au moins digénique.

Les formes familiales humaines d'hétérotaxie viscéro-auriculaire sont fréquentes et différents modes de transmission ont été décrits, autosomique dominant, récessif et récessif lié à l'X [32, 33]. Seul le gène responsable de l'hétérotaxie liée à l'X a été récemment cloné [34]. Il s'agit de ZIC3 (zinc finger protein of the cerebellum), codant pour une protéine à doigts de zinc. Elle interagit très probablement avec l'ADN pour régler la transcription de gènes cibles. La corrélation génotype/phénotype est intéressante puisque les hommes atteints ont un situs inversus incomplet avec des malformations cardiaques sévères et que les femmes hétérozygotes sont normales à l'exception d'une famille dans laquelle 3 des 9 femmes hétérozygotes ont un situs inversus totalis. La mutation inverserait le situs chez l'homme hémizygote et laisserait l'information en suspens chez la femme hétérozygote, la latéralisation se faisant au hasard.

Des mutations dans le gène codant pour la connexine 43, principale protéine constitutive des jonctions communiquantes cardiaques ont été rapportées dans des hétérotaxies viscéro-auriculaires. Plusieurs groupes

Figure 4. Les facteurs impliqués dans la latéralisation. En haut, les facteurs dont l'expression est la plus précoce, en bas les effecteurs potentiels au niveau de la matrice extracellulaire. Vg-1, Ird, ZIC3 et probablement inv ont une expression symétrique bien qu'ayant un rôle dans la latéralisation. 
ont rapidement infirmé cette découverte, très certainement liée à une erreur technique [35]. Cependant, l'invalidation du gène de la connexine 43 a relancé la polémique sur le rôle des connexions intercellulaires dans le cœur en développement puisque le phénotype de souris $C \times 43^{-1-}$ associe une atrésie pulmonaire à une soufflure de l'infundibulum $\mathrm{du}$ ventricule droit, mais la recherche de mutations chez les enfants ayant une atrésie pulmonaire à septum intact, phénotype similaire, a été négative. A ce jour, aucun modèle animal n'a permis d'identifier des mutations humaines dans les cardiopathies congénitales. Des perspectives viennent d'être ouvertes par la découverte de la régionalisation de l'expression des gènes cardiaques; certains de ceux-ci deviendront de bons gènes candidats.

\section{Maladie de Marfan}

Le substratum anatomique de la maladie de Marfan est une dégénérescence des fibres élastiques avec désorganisation des fibres collagènes. On sait aujourd'hui que ces lésions sont liées à la mutation du gène de la fibrilline 1 (FBN1) localisé sur le chromosome 15. FBN1 code pour la protéine constitutive majoritaire du tissu élastique: la fibrilline 1 qui est surtout présente dans les structures qui doivent résister aux charges et aux tensions (adventice aortique, ligament suspenseur du cristallin, peau) [36]; les mutations du gène codant pour la fibrilline 2 (FBN2) responsable de l'orientation de l'élastine et surtout présente dans le cartilage, la média aortique, les bronches sont responsables du syndrome congenital contractural arachnodactyly apparenté au syndrome de Marfan [37]. Les mutations de FBN1 sont très nombreuses et sont associées non seulement à la maladie de Marfan mais à d'autres fibrillinopathies: formes incomplètes, formes néonatales, ectopie cristallinienne, anévrismes isolés de l'aorte thoracique [38-40]. La répartition ubiquitaire de la fibrilline explique le pléiotropisme de la maladie de Marfan et permet d'en comprendre la sémiologie. La variabilité d'expression interfamiliale s'explique par l'hétérogénéité génétique (au moins deux gènes) [41] et allélique (mutations différentes de $F B N 1$ d'une famille à l'autre), d'où des formes modérées par réduction quantitative de fibrilline normale, et des formes graves par «dominance négative " dans lesquelles la fibrilline est anormale dans sa structure par altération des mécanismes de polymérisation. Le diagnostic biologique de fibrillinopathie peut se faire par un test protéique qui étudie la fibrilline sur une culture de fibroblastes du patient recueillis par biopsie cutanée. Pour l'heure, le diagnostic moléculaire visant à identifier la mutation dans le gène $F B N 1$ n'est pas réalisable en routine.

\section{Troubles de la conduction héréditaires}

Dans $95 \%$ des cas, l'étiologie des troubles de conduction est inconnue. Le risque d'avoir un bloc de conduction est multiplié par 2,5 chez les sujets apparentés aux patients ayant des troubles de la conduction isolés, le risque croissant avec l'âge. Ces troubles de conduction familiaux associent des hémiblocs de branche gauche, des blocs de branche droit complets ou incomplets, des blocs auriculo-ventriculaires de divers degrés, éventuellement une bradycardie sinusale mais pas de bloc de branche gauche. La transmission est autosomique dominante avec une pénétrance incomplète qui est nettement différente chez les hommes $(70 \%)$ et chez les femmes $(50 \%)$ [42]. Le début de la maladie est très variable et l'évolution progressive par paliers. Ces paliers apparaissent de manière imprévisible et expliquent le risque de malaise grave ou même de mort subite. Cette maladie est donc beaucoup moins bénigne qu'on ne pourrait l'imaginer. La mutation a été localisée en 19q13.3 [43,44] dans un intervalle de $2 \mathrm{cM}$ qui contient au moins 3 gènes candidats: le gène $B A X$ qui code pour une protéine qui forme un hétérodimère avec la protéine de BCL2, le gène HRC (histidine rich calcium binding protein), dont la protéine est synthétisée dans le cœur et qui fixe in vitro le calcium, enfin le gène $K C N A 7$ qui coderait pour un canal potassique. L'identification de ce gène permettra probablement de mettre en évidence un des facteurs de risque de la mort subite.
La cardiologie congénitale est une discipline clinique où les préoccupations du quotidien ressemblaient à des «problèmes de robinet". Les développements récents de la génétique moléculaire et les nouveaux horizons qui s'ouvrent depuis le début de la décennie ont modifié en profondeur la compréhension des mécanismes des malformations cardiaques. Demain, nous pourrons peut-être construire, en intégrant une anatomo-pathologie précise, jusqu'à l'histologie subcellulaire, les cascades d'événements en trois dimensions de la morphogenèse cardiaque et la connaissance des gènes exprimés aux différents endroits du cour en formation, une histoire différente de la genèse des cardiopathies

\section{Remerciements}

Les auteurs remercient l'Association Française contre les Myopathies, la Fondation de France, la SESERAC pour leur soutien.

\section{RÉFÉRENCES}

1. Kirby ML, Gale TF, Stewart DE. Neural crest cells contribute to aorticopulmonary septation. Science 1983 ; 220 : 1059-61.

2. Clark EB. Mechanisms in the pathogenesis of congenital heart defects. In : Pierpont ME, Moller JM, eds. The genetics of cardiovascular diseases. Boston: Martinus-Nijoff, $1986 ; 3-11$.

3. Nora JJ. Multifactorial inheritance hypothesis for the etiology of congenital heart diseases: the genetic environmental interaction. Circulation 1968; 38: 604-17.

4. Whittemore R, Wells JA, Castellsague X. A second-generation study of 427 probands with congenital heart disease and their 837 children. J Am Coll Cardiol 1994; 23: 1459-67.

5. Patterson DF, Pexieder T, Schnarr WR, Navratil T, Alaili R. A single major gene defect underlying cardiac cono-truncal malformations interferes with myocardial growth during embryonic development: studies in the CTD line of keeshond dogs. Am J Hum Genet 1993; 52: 388-97.

6. Brenner JL, Berg KA, Schneider DS, Clark EB, Boughman JA. Cardiac malformations in relatives of infants with hypoplastic left-heart syndrome. Am J Dis Child 1989; 143: 1492-4.

7. Glick BN, Roberts WC. Congenitally bicuspid aortic valve in multiple family members. Am J Cardiol 1994; 73 : 400-4. 


\section{RÉFÉRENCES}

8. Hurst JA, Hall CM, Baraitser M. The HoltOram syndrome. J Med Genet 1991; 28: 40610 .

9. Terret JA, Newbury-Ecob R, Cross GS, et al. Holt-Oram syndrome is a genetically heterogenous disease with one locus mapping to human chromosome 12q. Nat Genet 1994; 6 : 401-4.

10. Bonnet D, Pelet A, Legeai-Mallet L, et al. A gene for Holt-Oram syndrome maps to the distal long arm of chromosome 12. Nat Genet 1994; 6: 404-8.

11. Terrett JA, Newbury-Ecob R, Smith NM, et al. A translocation at $12 \mathrm{q} 2$ refines the interval containing the Holt-Oram syndrome 1 gene. Am J Hum Genet 1996; 59: 1337-42.

12. Li QY, Newbury-Ecob R, Terrett J, et al. Holt-Oram syndrome is caused by mutations in TBX5 a member of the Brachyury (T) gene family. Nat Genet 1997; 15: 21-9.

13. Gilgenkrantz S. Syndrome de SaethreChotzen et syndrome de Holt-Horam: implication de deux nouveaux gènes de développement TWIST et TBX5. Med Sci 1997; 13: 576-80.

14. Chessa M, Aggoun Y, Bonhoeffer P, Butera G, Lyonnet S, Bonnet D. Eterogenetica genica del Difetto Interatriale. Pediatria 1998 (sous presse).

15. Wilson L, Curtis A, Korenberg JR, et al. A large dominant pedigree of atrioventricular septal defect (AVSD): exclusion from the Down syndrome critical region on chromosome 21. Am J Hum Genet 1993; 53: 1262-8.

16. Bleyl S, Ruttenberg HD, Carey JC, Ward K. Familial total anomalous pulmonary venous return maps to chromosome 4 . Am J Hum Genet 1994; 52 : 462-6.

17. De la Chapelle A, Herva R, Koivisto M, Aula P. A deletion in chromosome 22 can cause DiGeorge syndrome. Hum Genet 1981; 57: 253-6.

18. Carey AH, Kelly D, Halford S, Wadey R, Wilson D, Goodship J, Burn J, Paul T, Sharkey A, Dumanski J, Nordenskjold MM, Williamson R, Scambler P. Molecular genetic study of the frequency of monosomy 22q11 in DiGeorge syndrome. Am J Hum Genet 1992; 51 : 964-70.

19. Bonnet D, Cormier-Daire V, Kachaner J, Szezepanski I, Souillard P, D, Munnich A,, Lyonnet S. Microsatellite DNA markers detect $95 \%$ of chromosome 22q11 deletions. Am J Med Genet 1997; 68: 182-4.

20. Demczuk S, Levy A, Aubry, et al. Excess of deletions of maternal origin in the DiGeorge/Velo-cardio-facial syndromes. A study of 22 new patients and review of the literature. Hum Genet 1995; 96: 9-13.

21. Augusseau S, Jouk PS, Jalbert P, Prieur $\mathrm{M}$. DiGeorge syndrome and $22 \mathrm{q} 11$ rearrangements. Hum Genet 1986; 74: 206.

22. Ryan AK, Goodship JA, Wilson DI, et al. 1052 interstitial chromosome 22q11 deletions: a European collaborative study. J Med Genet 1997; 34: 798-804.

23. Debrus S, Berger G, de Meeus A, Sauer U, Guillaumont S, Voisin M, Bozio A, Demczuk S, Aurias A, Bouvagnet P. Familial nonsyndromic conotruncal defects are not associated with a 22 q11 microdeletion. Hum Genet 1996; 97 : 138-44.

24. Budarf ML, Collins J, Gong W, Roe B, Wang Z, Bailey LC, Sellinger B, Michaud D, Driscoll DA, Emanuel BS. Cloning a balanced translocation associated with DiGeorge syndrome and identification of a disrupted candidate gene. Nat Genet 1995; 10: 269-78.

25. Levy A, Demczuk S, Aurias A, Depétris D, Mattei MG, Philip N. Interstitial 22q11 microdeletion excluding the ADU breakpoint in a patient with DiGeorge syndrome. Hum Mol Genet 1995; 4: 2417-9.

26. Chessa M, Butera G, Bonhoeffer P, Iserin L, Kachaner J, Lyonnet M, Munnich A, Sidi D, Bonnet D. Relationship of genotype 22q11 deletion to phenotype of pulmonary vessels in tetralogy of Fallot and pulmonary atresia-ventricular septal defect. Heart 1998; $79: 186-90$

27. Morris CA, Loker J, Ensing G, Stock AD. Supravalvular aortic stenosis cosegregates with a familial 6;7 translocation which disrupts the elastin gene. Am J Med Genet 1993; 46: 737-44.

28. Ewart AK, Morris CA, Atkinson D, Jin W, Sternes K, Spallone P, Stock AD, Leppert M, Keating MT. Hemizygosity at the elastin locus in a developmental disorder, Williams syndrome. Nat Genet 1993; 5 : 11-6.

29. Frangiskakis JM, Ewart AK, Morris CA, et al. LIM-kinase1 hemizygosity implicated in impaired visuospatial constructive cognition. Cell 1996; 86: 59-69.

30. Supp DM, Witte DP, Potter SS, Brueckner $M$. Mutation of an axonemal dynein affects left-right asymmetry in inversus viscerum mice. Nature 1997; 389: 963-6.

31. Yokoyama T, Copeland NG, Jenkins NA, Montgomery CA, Elder FFB, Overbeek PA. Reversal of left-right asymmetry: a situs inversus mutation. Science 1993; 260 : 679-82.

32. Alonso S, Pierpont ME, Radtke W, et al. Heterotaxia syndrome and autosomal dominant inheritance. Am J Med Genet 1995; 56 : $12-5$.

33. Debrus S, Sauer U, Gilgenkrantz S, Jost W, Jesberger HJ, Bouvagnet P. Autosomal recessive lateralization and midline defect: Blastogenesis recessive 1. Am J Med Genet 1997: 68: 401-4.

34. Gebbia M, Ferrero GB, Pilia G, et al. Xlinked situs abnormalities result from mutations in ZIC3. Nat Genet 1997; 17 : 305-8.

35. Gros D, Delorme B, Marics I, Théveniau-Ruissy M. Connexines et pathologie cardiaque : quelles connexions? Med Sci 1997; 13 : 1043-8.

36. Dietz HC, Cutting GR, Pyeritz RE, Maslen CL, Sakai LY, Corson GM, Puffenberg EG, Hamosh A, Nathankumar EJ, Curristin
SM. Marfan syndrome caused by a recurrent de novo mutation missense mutation in the fibrillin gene. Nature 1991; 352: 337-9.

37. Putnam EA, Zhang H, Ramirez F, Milewicz DM. Fibrillin-2 (FBN2) mutations result in the Marfan-like disorder, congenital contractural arachnodactyly. Nat Genet 1995; $11: 456-8$

38. Milewicz D, Michael K, Fisher N, Coselli J, Markello T, Biddinger A. Fibrillin-1 (FBN1) mutations in patients with thoracic aneurysms. Circulation 1996; 94: 2708-11.

39. Kainulainen K, Kattunen L, Puhakka L, Sakai L, Peltonen L. Mutations in the fibrillin gene responsible for dominant ectopia lentis and neonatal Marfan syndrome. Nat Genet 1994; 6: 64-9.

40. Collod G, Boileau C. Fibrillines et fibrillinopathies. Med Sci 1996; 12: 1077-86.

41. Collod G, Babron MC, Jondeau G, Coulon $\mathrm{M}$, Weissenbach J, Dubourg $\mathrm{O}$, Bourdarias JP, Bonaiti-Pelle C, Junien C, Boileau C. A second locus for Marfan syndrome maps to chromosomme 3p24.2-p25. Nat Genet $1994 ; 8: 264-8$

42. Stéphan E, de Meeus A, Bouvagnet P. Hereditary bundle branch defect: right bundle branch blocks of different causes have different morphologic characteristics. Am Heart J 1997; 133 : 249-56.

43. Brink PA, Ferreira A, Moolman JC, Hettie WW, van der Merwe PL, Corfield VA. Gene for progressive familial heart block type I maps to chromosome 19q13. Circulation 1995 ; 91 : 1633-40.

44. De Meeus A, Stéphan E, Debrus S, Jean MK, Loiselet J, Weissenbach J, Demaille J, Bouvagnet P. An isolated cardiac conduction disease maps to chromosome 19q. Circ Res 1996; 77 : 735-40.

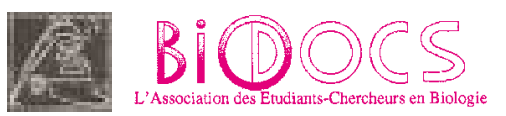

Vous voule 


\section{Summary}

Genetics of congenital

heart diseases

Recent progress has been made in the understanding of the mechanisms of congenital heart disease through new insights in molecular genetics in these congenital defects. Various approaches have been used towards the identification of genes responsible of cardiac malformations. Linkage analysis allowed the cloning of the Holt-Oram syndrome gene, $T B X 5$, and mapping of a gene for familial total anomalous pulmonary venous return on chromosome 4. Genetic heterogeneity of cardiac malformation is supported by the exclusion of the Holt-Oram syndrome genetic interval in isolated familial atrial septal defect which is the main cardiac defect in Holt-Oram syndrome. Cono-truncal diseases (tetralogy of Fallot, truncus arteriosus...) are frequently associated with chromosome 22q11 microdeletion. Physical mapping of the commonly deleted region allowed the identification of different genes potentially playing a role in the conal septation. Williams syndrome (facial dysmorphy, supravalvar aortic stenosis, mental retardation) is a contiguous gene syndrome due to a microdeletion on chromosome $7 q 23$ at the locus of the elastine gene and missense mutations have been found in non syndromic supravalvar aortic stenosis. Some clues have been found in animal models of congenital heart defects as lateralization defects associated with complex congenital heart malformations, and mutations in ZIC3 have recently been identified in X-linked heterotaxia. Fibrillin mutations were found responsible for Marfan syndrome in which genetic heterogeneity has also been demonstrated. Finally, a gene for familial isolated conduction defects was located on chromosome 19. Molecular genetics offers new perspectives in the understanding of congenital heart malformations etiology.

\section{TIRÉS À PART}

D. Bonnet.

$\mathrm{m} / \mathrm{s} n^{\circ} 10$, vol. 14 , octobre 98
Cent cinquantenaire de la SOCIÉTÉ D\&ßIOLOGIE Journée Claude Bernard 20 novembre 1998

Grand amphithéâtre du Collège de France

11, place Marcelin-Berthelot - 75005 Paris

\section{La Biologie, I'Homme et I'Éthique à I'aube du XXI siècle}

8 h 30 Accueil des participants - Introduction par Jacques PICARD, Président de la Société de Biologie

9 h 00 François JACOB, Prix Nobel, Membre de I'Institut Génétique et politique

9 h 40 Roger NORDMANN, Membre de l'Académie Nationale de Médecine 150 ans de la Société de Biologie

10 h 15 Axel KAHN, Membre de I'Institut De la fonction glycogénique du foie à la régulation des gènes par le glucose

$11 \mathrm{~h} 00$ Bernard JEANRENAUD, Professeur à I'Université de Genève De Claude Bernard aux relations entre I'hypothalamus et la périphérie et à leurs altérations dans l'obésité

11 h 40 Charles LEBLOND, Professeur à I'Université McGill, Montréal L'équilibre entre la synthèse et la lyse

12 h 30 LUNCH

14 h 00 Christian DE DUVE, Prix Nobel, Membre de l'Académie Royale de Médecine

Réflexion sur l'origine et l'évolution de la vie

14 h 30 Jacques DUMONT, Membre de l'Académie Royale de Médecine, Professeur à l'Université Libre de Bruxelles

Transduction des signaux et pathologie thyroïdienne : I'environnement et la génétique

15 h 10 Jacques RUFFIÉ, Membre de I'Institut Les défis de la science au début du troisième millénaire

15 h 50 Nicole LE DOUARIN, Membre de I'Institut Problèmes éthiques liés aux progrès de la biologie du développement

16 h 30 Georges DAVID, Membre de l'Académie Nationale de Médecine Les nouvelles procréations à l'origine d'une nouvelle biologie

17 h 10 Jean-Pierre CHANGEUX, Membre de I'Institut, Président du Comité National d'Éthique

Réflexions d'un neurobiologiste sur les fondements de l'éthique: de I'ontologie à la déontologie

17 h 45 Conclusion par Jacques POLONOVSKI, Membre de l'Académie Nationale de Médecine, Secrétaire général de la Société de Biologie

\section{Une séance de communications aura lieu le 19 novembre 1998} de $14 \mathrm{~h} 00$ à $18 \mathrm{~h} 00$ avec la participation de :

Harold KALANT (Toronto), Christian DOUTREMÉPUICH (Bordeaux), B.A. Denian (Pékin), CHEN Zhu (Shanghaï) Susan HOLLÁN (Budapest), Gustav BORN (Londres), Hannes STÄHELIN (Bâle), Francis KARST (Poitiers)

Renseignements et inscription pour le lunch avant le 15/10/98 auprès du Secrétariat de la Société de Biologie,

3, rue d'UIm - 75231 PARIS cedex 05 - ๔ ou Fax : 0144271340 